\title{
A murine model of NKT cell-mediated liver injury induced by alpha-galactosylceramide/D-galactosamine
}

\section{Hideki Fujii • Shuichi Seki • Sawako Kobayashi • \\ Takuya Kitada • Nobuyoshi Kawakita • Keishi Adachi • Hiroko Tsutsui • Kenji Nakanishi • Hiromi Fujiwara • \\ Yoshinori Ikarashi • Masaru Taniguchi • \\ Mitchell Kronenberg • Masaru Ikemoto • \\ Yuji Nakajima Tetsuo Arakawa $\cdot$ Kenji Kaneda}

Published online: 4 August 2006

(C) Springer-Verlag 2006

The online version of the original article can be found at: http://dx.doi. org/10.1007/s00428-005-1265-8

H. Fujii $(\bowtie) \cdot$ S. Seki $\cdot$ S. Kobayashi $\cdot$ T. Kitada $\cdot$ N. Kawakita Departmet of Hepatology,

Osaka City University Graduate School of Medicine,

1-4-3 Asahimachi, Abeno-ku,

Osaka 545-8585, Japan

e-mail: rolahideki@med.osaka-cu.ac.jp

H. Fujii $\cdot$ M. Ikemoto $\cdot$ Y. Nakajima $\cdot$ K. Kaneda

Departmet of Anatomy,

Osaka City University Graduate School of Medicine,

Osaka, Japan

\section{K. Adachi $\cdot$ H. Tsutsui $\cdot$ K. Nakanishi}

Core Research for Evolutional Science and Technology,

Japan Science and Technology Agency,

Kawaguchi, Japan

H. Tsutsui $\cdot$ K. Nakanishi

Departmet of Immunology and Medical Zoology,

Hyogo College of Medicine,

Nishinomiya, Japan

H. Fujiwara

Biomedical Research Center,

Osaka University Graduate School of Medicine,

Suita, Japan

\section{Y. Ikarashi}

Pharmacology Division,

National Cancer Center Research Institute,

Tokyo, Japan
Virchows Arch (2005) 446:663-673

The name of the twelveth author is Mitchell Kronenberg not Kronenberg Mitchell.

M. Taniguchi

Institute of Physical and Chemical Research, Research Center for Allergy and Immunology,

Tokyo, Japan

M. Taniguchi

Chiba University Graduate School of Medicine,

Chiba, Japan

M. Kronenberg

La Jolla Institute for Allergy and Immunology,

San Diego, CA, USA

T. Arakawa

Department of Gastoroenterology,

Osaka City University Graduate School of Medicine,

Osaka, Japan 